\title{
Questionnaire Overview
}

\begin{tabular}{||lll||}
\hline Baseline (Day 1) & $>$ & Quality of Work \\
& $>$ & Mood States \\
& $>$ & Student behavior \\
Ongoing & $>$ & Adherence to standing protocol \\
& $>$ & Quality of Work (daily) \\
Follow-up (Day 40) & $>$ & Mood States (daily) \\
& $>$ & Adherence to standing protocol (daily) \\
& $>$ & Quality of Work \\
& $>$ & Mood States \\
& $>$ & Adhent behavior \\
& $>$ & Attitudes regarding Sit-to-Stand devices (intervention group) \\
\hline
\end{tabular}

